\title{
Developing Gradiometer to Reduce the Low Frequency Noise of Magnetic Sensor with Amorphous Wire
}

\author{
Dongfeng He \\ National Institute for Materials Science, 1-2-1 Sengen, Tsukuba, Ibaraki, Japan
}

Email address:

He.dongfeng@nims.go.jp

To cite this article:

Dongfeng He. Developing Gradiometer to Reduce the Low Frequency Noise of Magnetic Sensor with Amorphous Wire. Journal of Electrical and Electronic Engineering. Vol. 9, No. 2, 2021, pp. 33-40. doi: 10.11648/j.jeee.20210902.11

Received: March 29, 2021; Accepted: April 13, 2021; Published: April 23, 2021

\begin{abstract}
Pico-Tesla level high sensitivity magnetic sensor has been developed using FeCoSiB amorphous wire as the core of a coil, but the low frequency noise of the magnetic sensor is somewhat high, and some applications of magnetic sensors, such as magnetic microscope or deep defect detection, require that the sensors have low noise at low frequency. To reduce the noise, it is necessary to know the noise sources of the magnetic sensor. By measuring the noise spectrum of each part of the magnetic sensor with $\mathrm{FeCoSiB}$ amorphous wire was measured, it was found that the white noise of the magnetic sensor was mainly determined by the noise of the preamplifier and the low frequency noise was mainly determined by the low frequency noises of the DC bias current and $\mathrm{AC}$ bias current of the $\mathrm{FeCoSiB}$ magnetic sensor. To reduce the low frequency noise of the magnetic sensor caused by the bias current noise, an electrical gradiometer composed of two magnetic sensors sharing the same DC bias current and AC bias current was developed. The results proved that the gradiometer was not only effective to reduce the low frequency noise caused the bias current, but also effective to reduce the environmental noise caused by the $50 \mathrm{~Hz}$ line interference. Using the gradiometer, an eddy current testing (ECT) system were constructed, and the deep defect with the depth of about $8 \mathrm{~mm}$ in an aluminum plate was successfully detected by the ECT system.
\end{abstract}

Keywords: Magnetic Sensor, FeCoSiB, Amorphous Wire, Noise, Gradiometer, Eddy Current Testing

\section{Introduction}

Room temperature pT-level high sensitivity magnetic sensors have been developed [1-5]. Although these sensors are not so sensitive compared with superconducting quantum interference device (SQUID) and on optical pumping magnetic sensor $[6,7]$, due to the small sizes and the easy operation, room temperature high-sensitivity magnetic sensors have found applications of bio-magnetic sensing [8], communication [9], nondestructive evaluation $[10,11]$ and automotive [12]. For some applications of magnetic sensors, such as the magnetic microscope [13] or the deep defect detection using eddy current testing (ECT) method [14], magnetic sensor with low noise at low frequency is needed. For ECT method, low frequency has big penetration depth, and is used to detect deep defect. To reduce the noise of the magnetic sensor, the investigations of the dependence of noises are important $[15,16]$.

The high-sensitivity magnetic sensors using a coil with the $\left(\mathrm{Fe}_{0.06} \mathrm{Co}_{0.94}\right)_{72.5} \mathrm{Si}_{2.5} \mathrm{~B}_{15}(\mathrm{FeCoSiB})$ amorphous wire as the core were reported [17-19] and the white noise spectrum of about $6 \mathrm{pT} / \sqrt{ } \mathrm{Hz}$ was achieved. Different from normal MI sensors, the amorphous wire of this kind of magnetic sensor had no any electrical connection with the cable. A DC current and an $\mathrm{AC}$ current were used to supply the bias magnetic fields to the sensor through the coil coupled with the amorphous wire. When the DC bias magnetic field was adjusted near to the saturation magnetization point of the amorphous wire, the inductance of the coil changed with the applied magnetic field, then the amplitude of the AC signal also changed with magnetic field. After a demodulator, the output changed with magnetic field. For this magnetic sensor, only one coaxial cable was used to connect the sensing element and the driving circuit. The simple structure of this magnetic sensor was useful for some industrial applications. But the low frequency noise of this kind of magnetic sensor was higher than normal MI sensors. To further improve the magnetic field resolution of the magnetic sensor, it is necessary to know the noise source of the magnetic sensor with $\mathrm{FeCoSiB}$ amorphous wire. 
This kind of magnetic sensor includes high frequency circuit and low frequency circuit. It is not easy to determine the noise contribution of each part of the sensor system. In this paper, the steps to determine the noise contribution of each part of the sensor system are given. And an electrical gradiometer was developed to reduce the low frequency noise of the magnetic sensor. Different from normal electrical gradiometer [20-22] with independent driving circuits for two sensors, the two sensors of this gradiometer shared the $\mathrm{DC}$ bias current and the AC bias current.

\section{Measuring the Noise of Each Part of the Magnetic Sensor}

\subsection{Block Diagram of the Magnetic Sensor with FeCoSiB Amorphous Wire}

Figure 1 shows the schematic block diagram of the magnetic sensor with the ferromagnetic amorphous wire of FeCoSiB. It measured the vector magnetic field along the direction of the amorphous wire. The $\mathrm{FeCoSiB}$ amorphous wire with the diameter of $0.1 \mathrm{~mm}$ and the length of $5 \mathrm{~mm}$ was used. The coil wound round the amorphous wire was 30 turns using the cooper wire of $\phi 0.1 \mathrm{~mm}$. The outer diameter and the inner diameter of the coil were about $1.5 \mathrm{~mm}$ and $0.5 \mathrm{~mm}$ respectively. The magnetic sensor was biased by a DC current of about $50 \mathrm{~mA}$ and an $\mathrm{AC}$ current of $1 \mathrm{MHz}$ with the amplitude of about $10 \mathrm{~mA}$. To isolate the DC bias current, the $\mathrm{AC}$ bias current and the signal, the inductor $\mathrm{L}_{\mathrm{D}}$ and the capacitors $\mathrm{C} 1, \mathrm{C} 2$ were used in the circuit. To increase the signal amplitude, a resonant circuit, which was composed of a capacitor $\mathrm{C}$ and an inductor $\mathrm{L}$, was used. With the resonant circuit, the signal amplitude was increased by about 10 times. By adjusting the DC current, the amorphous wire was biased near the saturation magnetization, then the $1 \mathrm{MHz}$ signal amplitude of the coil changed with the applied magnetic field. The demodulator, composed of a diode, a $1 \mathrm{k} \Omega$ resistor and a capacitor $\mathrm{C} 3$, was used get the amplitude of the $1 \mathrm{MHz}$ signal. The preamplifier for the $1 \mathrm{MHz}$ signal was made by AD829 and the gain was about $20 \mathrm{~dB}$. The low frequency amplifier after the demodulator was made by an operation amplifier of OP275 and the gain of the AMP was about $46 \mathrm{~dB}$. The best magnetic field resolution of about $6 \mathrm{pT} / \sqrt{\mathrm{Hz}}$ was achieved for the magnetic sensor with the optimized parameters. In this paper, easily achieved $9 \mathrm{pT} / \sqrt{ } \mathrm{Hz}$ magnetic sensors were used.

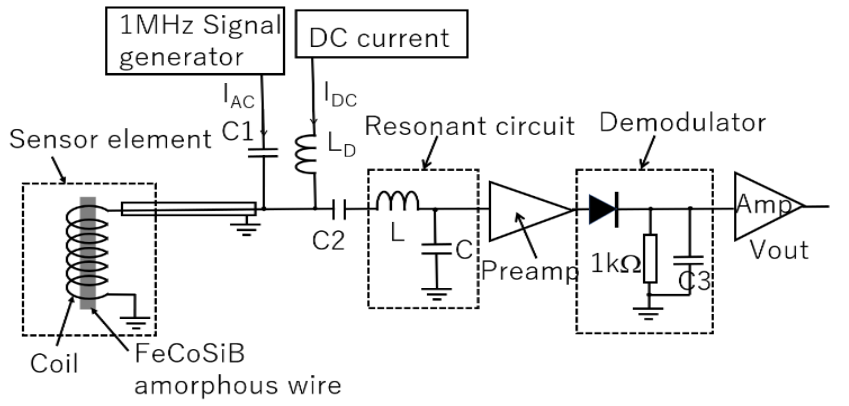

Figure 1. Block diagram of the magnetic sensor with amorphous wire.

\subsection{Total Voltage Noise Spectrum of the Magnetic Sensor}

The noises produced by the amorphous wire, the AC current, the DC current, the preamplifier, and the amplifier all have contributions to the total output signal noises of the magnetic sensor. From my experiment, the diode for the demodulator has less contribution to the total noise, so the total voltage noise spectrum of the magnetic sensor can be expressed by following equation:

$$
V_{N}=\sqrt{V_{N, \text { Wire }}^{2}+V_{N, A C}^{2}+V_{N, D C}^{2}+V_{N, \text { Preamp }}^{2}+V_{N, A m p}^{2}}
$$

Where, $V_{N}$ is the total output voltage noise spectrum of the magnetic sensor. $\mathrm{V}_{\mathrm{N} \text {, Wire }}$ is the noise produced by the $\mathrm{FeCoSiB}$ amorphous wire. $\mathrm{V}_{\mathrm{N}, \mathrm{AC}}$ is the noise contribution caused by the AC bias current noise. $\mathrm{V}_{\mathrm{N}, \mathrm{DC}}$ is the noise contribution caused by the $\mathrm{DC}$ bias current noise, $\mathrm{V}_{\mathrm{N} \text {,Preamp }}$ is the noise contribution by the preamplifier. $\mathrm{V}_{\mathrm{N} \text {,Amp }}$ is the noise contribution by the amplifier. The noise contributions of the Amp, DC bias current, $\mathrm{AC}$ bias current, preamplifier and the sensor element could be measured. A vector signal analyzer of HP89441A was used to measure noise spectrums. To reduce the influence of the environmental noise, the measurements were done in a 3-layer permalloy shielding box.

Figure 2 shows the total voltage noise spectrum of the magnetic sensor with $\mathrm{FeCoSiB}$ amorphous wire. The white noise of the output signal was about $10 \mu \mathrm{V} / \sqrt{ } \mathrm{Hz}$ and the low frequency noise at $2 \mathrm{~Hz}$ was about $45 \mu \mathrm{V} / \sqrt{ } \mathrm{Hz}$.

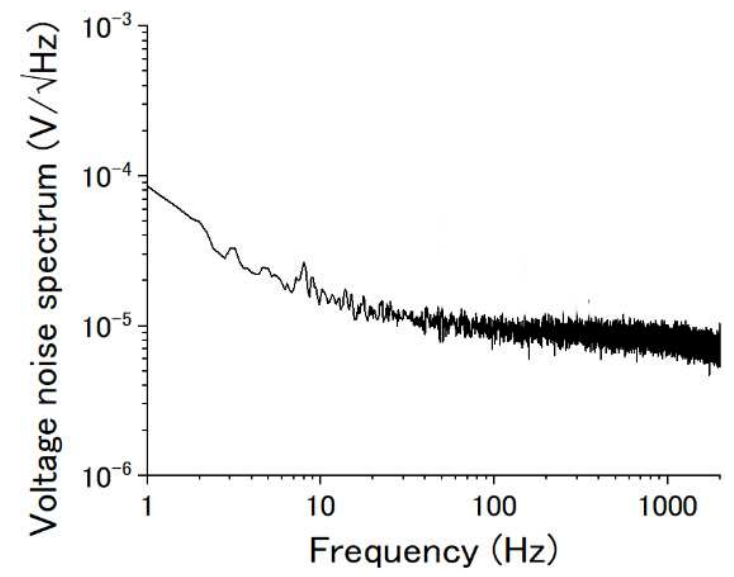

Figure 2. Total voltage noise spectrum of the output signal of the magnetic sensor with $\mathrm{FeCOSiB}$ amorphous wire.

\subsection{Noise Spectrums at Different Conditions}

To measure the noises caused by the AC current and DC current, amplifier is necessary, so firstly, the noise produced by the amplifier was measured. Figure 3(a) shows the method to measure the noise produced by the amplifier. The Amp was made of OP275 and the gain of the Amp was about 46 $\mathrm{dB}$. A resistor of $1 \mathrm{k} \Omega$ was connected to the input of the Amp, which was similar with the real circuit. Figure $3(\mathrm{~b})$ shows the voltage noise spectrum of the output of the Amp. The white noise of the output signal was about $1.5 \mu \mathrm{V} / \sqrt{ } \mathrm{Hz}$ and the low frequency noise at $2 \mathrm{~Hz}$ was about $8.8 \mu \mathrm{V} / \sqrt{ } \mathrm{Hz}$. 


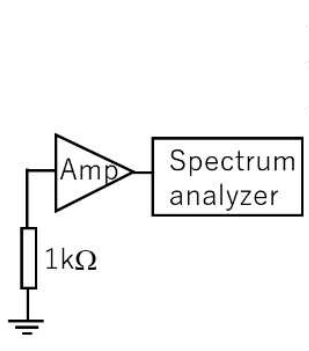

(a)

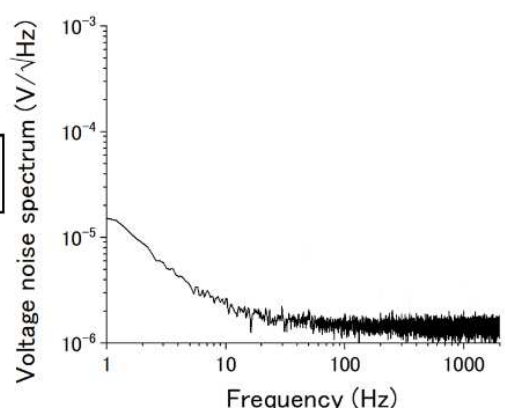

(b)
Figure 3. (a). Method to measure the noise spectrum of the Amp. (b). Voltage noise spectrum of the Amp output signal caption of the figure.

The DC bias current of the magnetic sensor was supplied by a DC voltage through a resistor. It is an important noise source of magnetic sensor. The DC voltage may make the Amp overload. To measure the noise from the DC power, a capacitor is normally needed to isolate the DC voltage [23]. Figure 4(a) shows the method to measure the noise spectrum of the $5 \mathrm{~V}$ DC voltage. A big capacitor of $220 \mu \mathrm{F}$ was used to isolate the DC voltage, the cut off frequency of the CR high pass filter was about $0.7 \mathrm{~Hz}$. Figure 4(b) shows noise spectrum of the output signal. The white noise of the output signal was about $2.3 \mu \mathrm{V} / \sqrt{ } \mathrm{Hz}$ and the low frequency noise at $2 \mathrm{~Hz}$ was about $70 \mu \mathrm{V} / \sqrt{ } \mathrm{Hz}$.

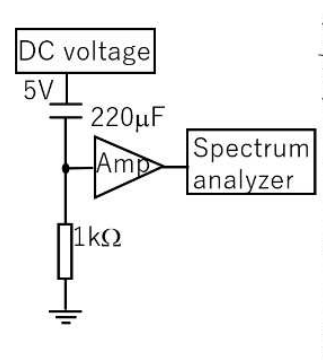

(a)

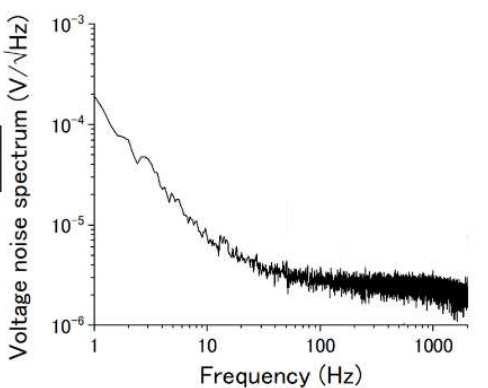

(b)
Figure 4. (a). Method to measure the noise spectrum of the DC voltage. (b). Voltage noise spectrum of the output signal.

The $1 \mathrm{MHz}$ AC bias current of the magnetic sensor was supplied by a $1 \mathrm{MHz}$ signal generator through a resistor. To measure the noise caused by the amplitude fluctuation of the $1 \mathrm{MHz}$ AC bias current. A diode with the low forward voltage of about $0.25 \mathrm{~V}$ was used to change the amplitude of the AC voltage to a DC voltage. Figure 5(a) shows the method to measure the noise spectrum of the $1 \mathrm{MHz} \mathrm{AC}$ voltage. If the $\mathrm{AC}$ voltage is bigger enough, the working point of the diode has less influence. In this experiment, the amplitude of the AC voltage was $\pm 5 \mathrm{~V}$. Two resistors of 330 $\Omega$ were used and the amplitude of the AC voltage to the diode was about was $\pm 2.5 \mathrm{~V}$, which was similar with the real amplitude of magnetic sensor. A low pass filter (LPF) was used after the diode and the cut off frequency of the LPF was about $6 \mathrm{kHz}$. Figure 5(b) shows the noise spectrum of the output signal. The white noise of the output signal was about $2.1 \mu \mathrm{V} / \sqrt{\mathrm{Hz}}$ and the low frequency noise at $2 \mathrm{~Hz}$ was about $18 \mu \mathrm{V} / \sqrt{ } \mathrm{Hz}$.
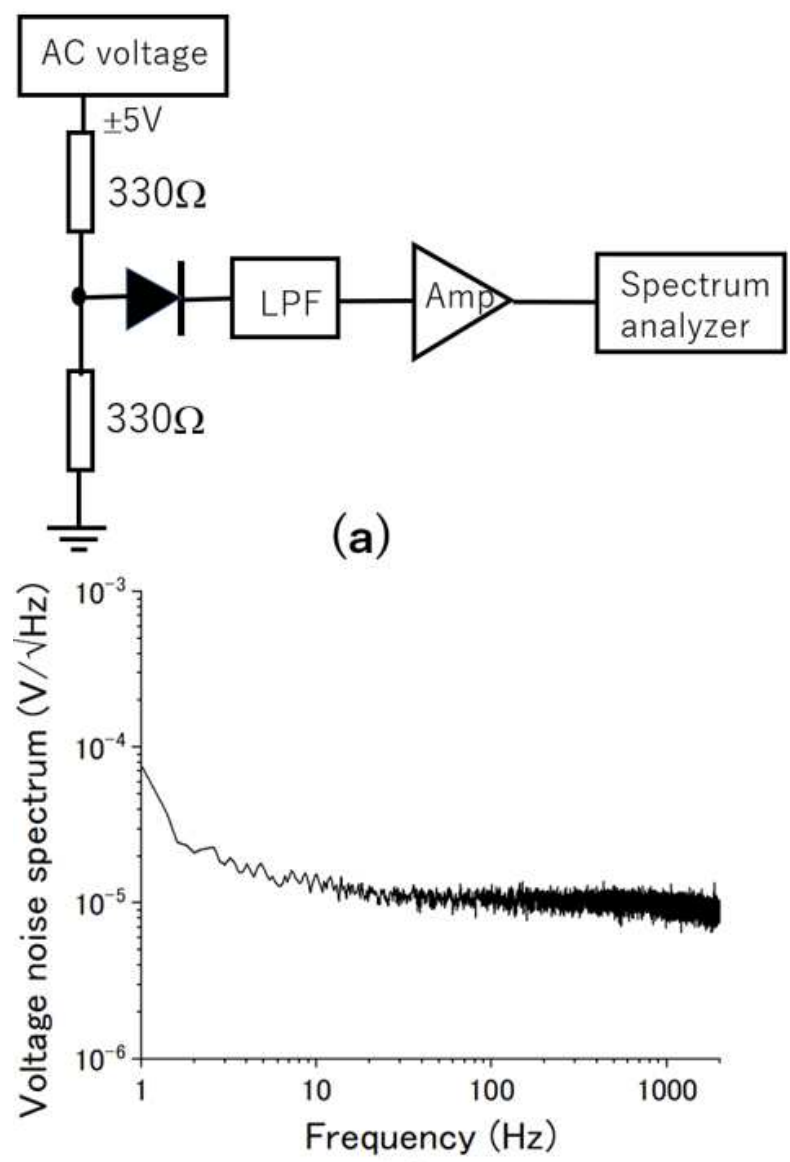

(b)

Figure 5. (a). Method to measure the noise spectrum of the $A C$ voltage. (b). Voltage noise spectrum of the output signal.

To measure the noise contribution of the preamplifier, the preamplifier was connected to an inductor of $2 \mu \mathrm{H}$, which was similar with the inductance of the magnetic sensor with amorphous wire. Figure 6(a) shows the method. The AC bias current was kept to be same as that of the real magnetic sensor, but the DC bias current was adjusted to zero. Figure 6(b) shows the noise spectrum of the output signal with the $2 \mu \mathrm{H}$ inductor. The white noise of the output signal was about 10 $\mu \mathrm{V} / \sqrt{\mathrm{Hz}}$ and the low frequency noise at $2 \mathrm{~Hz}$ was about 21 $\mu \mathrm{V} / \sqrt{ } \mathrm{Hz}$.

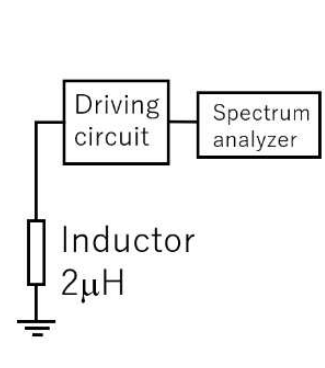

(a)

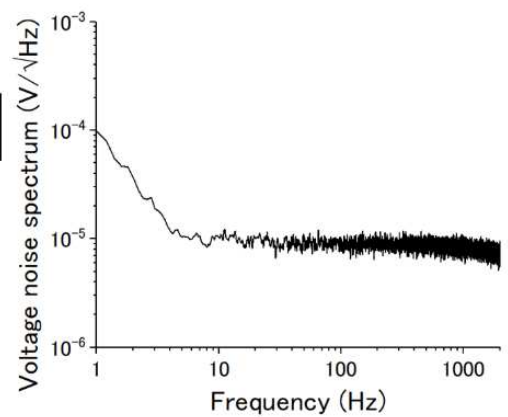

(b)
Figure 6. (a). Method to measure the noise spectrum of the preamplifier. (b). Voltage noise spectrum of the output signal. 


\subsection{Noise Contribution of Each Part of the Magnetic Sensor}

The contributions of the noise of each part of the magnetic sensor system to the total output noise were calculated. For the noise contribution of the DC bias, the DC bias voltage produced a noise current through a resistor, then produced a noise of magnetic field through the coil wound around the amorphous wire and caused the noise of the output signal. The equation (2) was used to calculate its contribution to the total noise.

$$
V_{D C}=k_{1} \cdot \sqrt{V_{D C A m p}^{2}-V_{A m p}^{2}}
$$

Where, $V_{D C A m p}$ was the noise spectrum of the DC bias voltage shown in Figure 4(b), $V_{A m p}$ was the noise spectrum of the Amp shown in Figure 3(b), $k_{l}$ was a factor changing the DC bias voltage to the output voltage. The measured value of $k_{l}$ was about 0.45 . The noise spectrum of $V_{D C A m p}$ included the noise of the DC bias voltage and the noise of the Amp, so equation (2) was used to calculate the noise spectrum of $V_{D C}$. Figure 7(a) shows the calculated noise spectrum of $V_{D C}$ Using equation (2). The white noise at $200 \mathrm{~Hz}$ was about 1 $\mu \mathrm{V} / \sqrt{\mathrm{Hz}}$. The low frequency noise was about $2 \mu \mathrm{V} / \sqrt{ } \mathrm{Hz}$ at 20 $\mathrm{Hz}$ and $31 \mu \mathrm{V} / \sqrt{ } \mathrm{Hz}$ at $2 \mathrm{~Hz}$.

The contribution of the AC bias current was calculated by the equation (3). Where, $V_{A C A m p}$ was the noise spectrum of AC bias voltage shown in Figure 5(b), $V_{A m p}$ was the noise spectrum of the Amp shown in Figure 3(b), $k_{2}$ was a factor to represent the ratio of the real $\mathrm{AC}$ signal amplitude of the magnetic sensor and the AC signal amplitude used to measure the noise spectrum. In our experiment, $k_{2}$ was equal to 1 , because the measured AC signal amplitude was chosen to be similar with the real AC signal amplitude of the magnetic sensor. Figure 7(b) shows the calculated noise spectrum of $V_{A C}$. The white noise at $200 \mathrm{~Hz}$ was about 1.7 $\mu \mathrm{V} / \sqrt{ } \mathrm{Hz}$. The low frequency noise was about $1.7 \mu \mathrm{V} / \sqrt{\mathrm{Hz}}$ at $20 \mathrm{~Hz}$ and $16 \mu \mathrm{V} / \sqrt{ } \mathrm{Hz}$ at $2 \mathrm{~Hz}$.

$$
V_{A C}=k_{2} \cdot \sqrt{V_{A C A m p}^{2}-V_{A m p}^{2}}
$$

The contribution of the preamplifier was calculated by equation (4). Where, $V_{A C A m p}$ was the noise spectrum of AC bias voltage shown in Figure $5(\mathrm{~b}), V_{\text {PreampACAmp }}$ was the total noise spectrum of the preamplifier, $\mathrm{AC}$ bias and the Amp, shown in Figure 6(b). Figure 7(c) shows the calculated noise spectrum of the preamplifier $V_{\text {Preamp }}$. The white noise at 200 $\mathrm{Hz}$ was about $9 \mu \mathrm{V} / \sqrt{ } \mathrm{Hz}$. The low frequency noise was about $10 \mu \mathrm{V} / \sqrt{ } \mathrm{Hz}$ at $20 \mathrm{~Hz}$ and $31 \mu \mathrm{V} / \sqrt{ } \mathrm{Hz}$ at $2 \mathrm{~Hz}$.

$$
V_{\text {Preamp }}=\sqrt{V_{\text {PreampACAmp }}^{2}-V_{A C A m p}^{2}}
$$
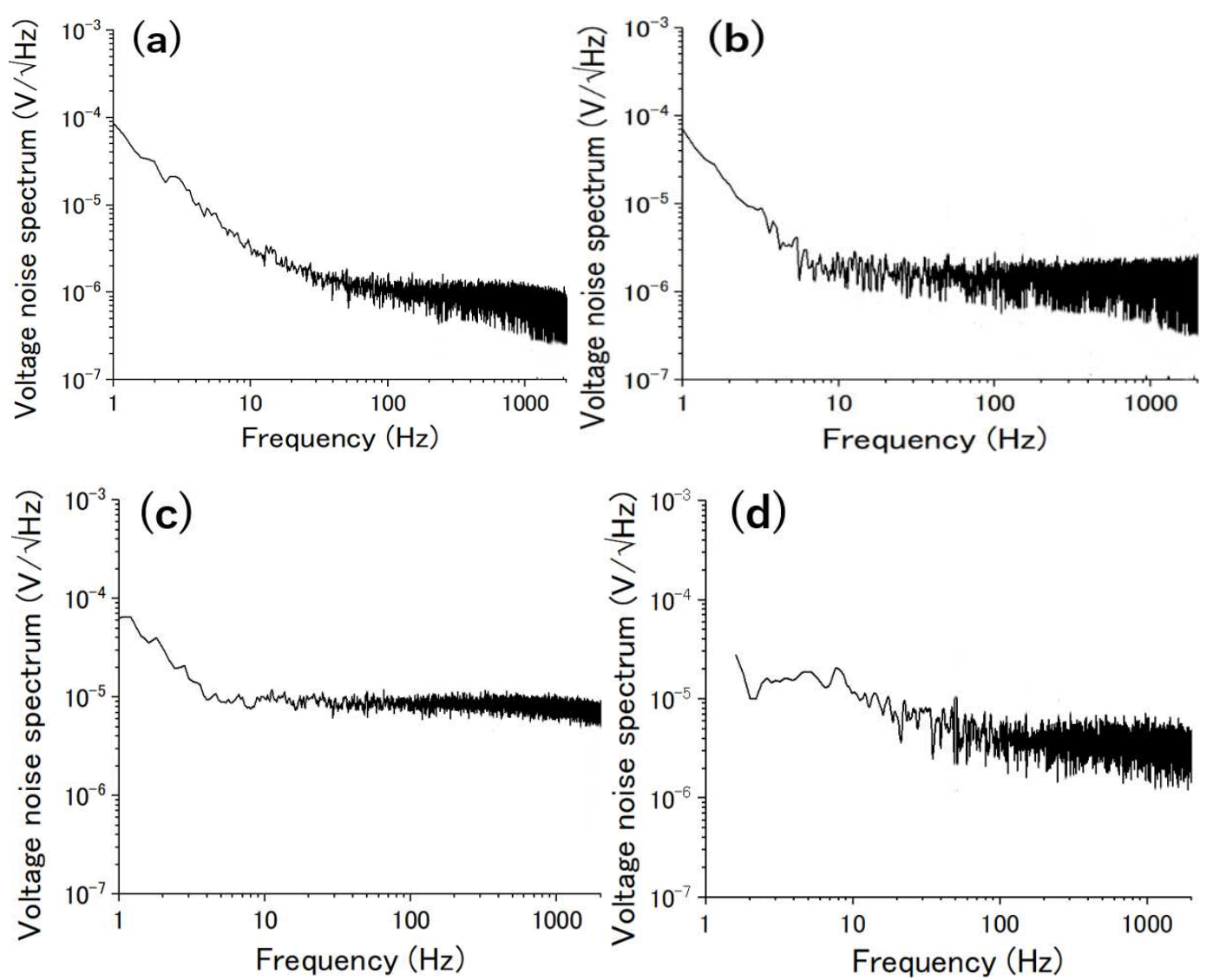

Figure 7. (a). Calculated voltage noise spectrum produced by the DC bias current. (b). Calculated voltage noise spectrum produced by the AC bias current. (c). Calculated voltage noise spectrum produced by the preamplifier. (d). Calculated voltage noise spectrum produced by the FeCoSiB amorphous wire. 
The contribution of the amorphous wire to total noise of the sensor was calculated by equation (5). Where, $V_{\text {PreampACAmp }}$ was the total noise spectrum of the preamplifier, $\mathrm{AC}$ bias and the Amp, shown in Figure 6(b), $V_{D C}$ was the noise spectrum caused by the DC bias current, shown in Figure 7(a), Vsensor was the total noise spectrum of the sensor shown in Figure 1. Figure 7(d) shows the calculated noise spectrum of $V_{\text {Wire }}$. The white noise at $200 \mathrm{~Hz}$ was about $4 \mu \mathrm{V} / \sqrt{\mathrm{Hz}}$. The low frequency noise was about $6.6 \mu \mathrm{V} / \sqrt{ } \mathrm{Hz}$ at $20 \mathrm{~Hz}$ and $14 \mu \mathrm{V} / \sqrt{ } \mathrm{Hz}$ at $2 \mathrm{~Hz}$.

$$
V_{\text {Wire }}=\sqrt{V_{\text {Sensor }}^{2}-V_{\text {PreampACAmp }}^{2}-V_{D C}^{2}}
$$

Table 1 lists the noise contribution of each part of magnetic sensor to the total noise at the frequency of $2 \mathrm{~Hz}$, $20 \mathrm{~Hz}$ and $200 \mathrm{~Hz}$ (white noise). Because of the low frequency Amp was at the last stage of the driving circuit, it had smallest contribution to the total noise of the magnetic sensor. For the white noise (at $200 \mathrm{~Hz}$ ). The preamplifier had the biggest contribution to the total noise of the magnetic sensor. For the low frequency noise at $20 \mathrm{~Hz}$, the noises of the preamplifier and the $\mathrm{FeCoSiB}$ amorphous wire had bigger contributions than those of the DC bias current and the AC bias current. For the low frequency noise at $2 \mathrm{~Hz}$, the noises of the DC bias current and the preamplifier had bigger contributions than those of the $\mathrm{AC}$ bias current and the FeCoSiB amorphous wire.

According to the experimental results, the signal amplitude should be increased to reduce the white noise of the magnetic sensor, and decrease the low frequency noise of the DC bias current to reduce the low frequency noise of the magnetic sensor

Table 1. Voltage noise spectrum of each part of the magnetic sensor.

\begin{tabular}{llll}
\hline & $\begin{array}{l}\text { Noise at } 2 \mathrm{~Hz} \\
(\boldsymbol{\mu} \mathbf{V} / \sqrt{\mathbf{H z}})\end{array}$ & $\begin{array}{l}\text { Noise at } 20 \mathrm{~Hz} \\
(\boldsymbol{\mu} / \sqrt{ } \mathbf{H z})\end{array}$ & $\begin{array}{l}\text { Noise at } 200 \mathrm{~Hz} \\
(\boldsymbol{\mu} \mathbf{V} / \sqrt{\mathbf{H z})}\end{array}$ \\
\hline Amp & 8.8 & 1.8 & 1.5 \\
$\mathrm{I}_{\mathrm{DC}}$ & 31 & 2 & 1 \\
$\mathrm{I}_{\mathrm{AC}}$ & 15 & 1.7 & 1.7 \\
Preamp & 31 & 10 & 9 \\
FeCoSiB wire & 14 & 6.6 & 4 \\
\hline
\end{tabular}

\section{Developing Gradiometer to Reduce the Low Frequency Noise of the Magnetic Sensor}

\subsection{Setup of the Gradiometer}

Electrical gradiometer is commonly used to reduce the environmental noise when the magnetic sensor is used in unshielded environment [20,21]. Figure 8 shows the principle of the electrical gradiometer composed of the two magnetic sensors. The output of the gradiometer is the subtraction of the outputs of the magnetic sensor 1 and the magnetic sensor 2 . The environmental noise produces the same outputs for the two magnetic sensors, so it is eliminated after the subtraction.

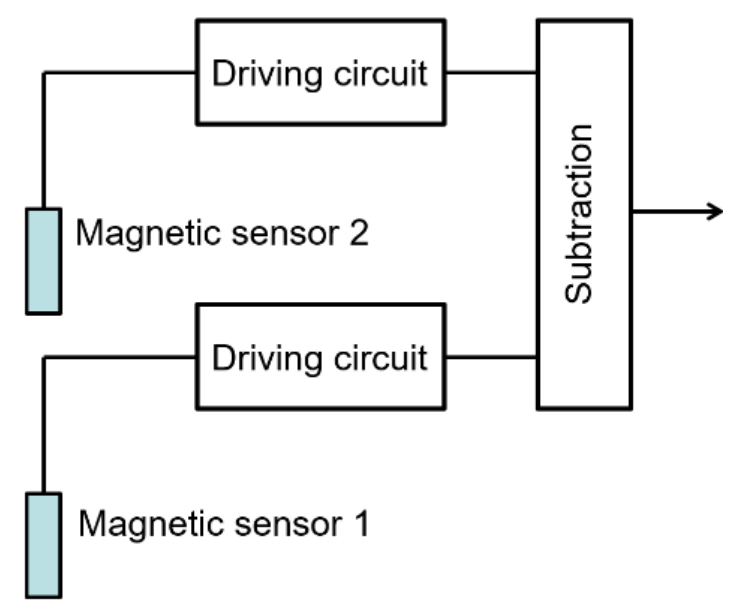

Figure 8. Electrical gradiometer composed of two magnetic sensors.

The electrical gradiometer was used to reduce the influence of the noise of the bias current for our magnetic sensor. Figure 9 shows the schematic block diagram of the electrical gradiometer composed of two magnetic sensors. Different from normal electrical gradiometer, the two magnetic sensors in our gradiometer shared the same bias DC current and AC current. The second magnetic sensor was used as a reference sensor to reduce the noise contribution of the bias current.

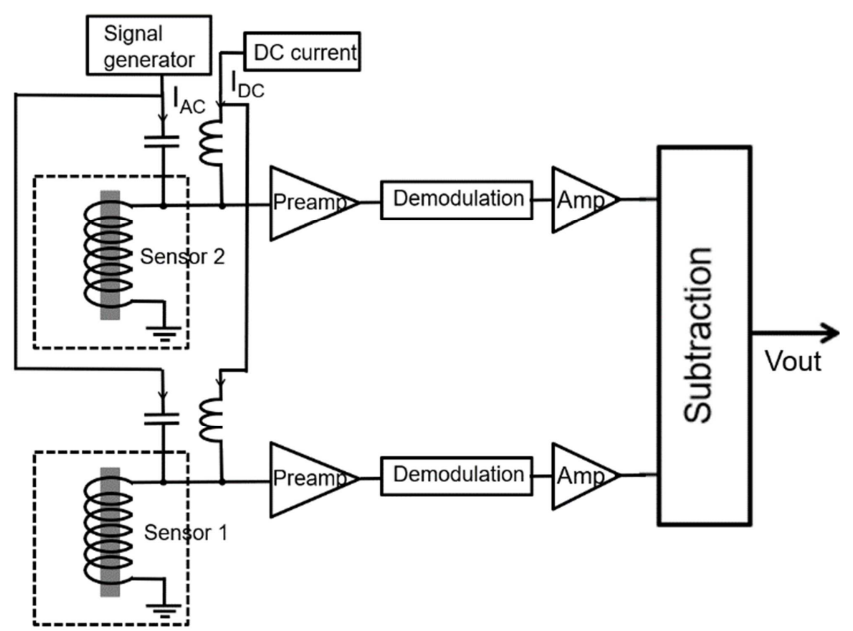

Figure 9. Schematic block diagram of the electrical gradiometer composed of two magnetic sensors sharing the same DC bias current and AC bias current.

Two similar sensors made of FeCoSiB amorphous wire were used. The distance between the two sensors was about 5 $\mathrm{cm}$. The DC bias current and the AC bias current were used to bias the magnetic sensor 1 and the magnetic sensor 2. Each sensor had its own preamplifier, demodulation, and the amplifier. The output of the gradiometer was the subtraction of the outputs of the two magnetic sensors. Because the sensor 1 and sensor 2 were biased by the same DC bias current and the AC bias current, the noises of the bias currents caused the similar outputs of the sensor 1 and the sensor 2. After the subtraction, the influence of the noises of the bias currents could be eliminated. 


\subsection{Noise Spectrum of the Gradiometer}

To measure the magnetic field noise spectrum of the magnetic sensor, a Helmholtz coil was used to do the calibration. The output voltage of the gradiometer for the applied magnetic field with the amplitude of $1 \mu \mathrm{T}$ was measured. It was about $1.05 \mathrm{~V} / \mu \mathrm{T}$. Using this value, the magnetic field noise spectrums could be easily calculated from the output voltage noise spectrum of the magnetic sensor. Figure 10 shows the magnetic field noise spectrums of the magnetic sensor and the gradiometer measured in a magnetic shielding box. Compared with the magnetic field noise spectrum of the magnetic sensor, the gradiometer had lower noise at low frequency. This result proved that It was effective for the gradiometer to reduce the low frequency noise caused by the bias DC current or AC current.
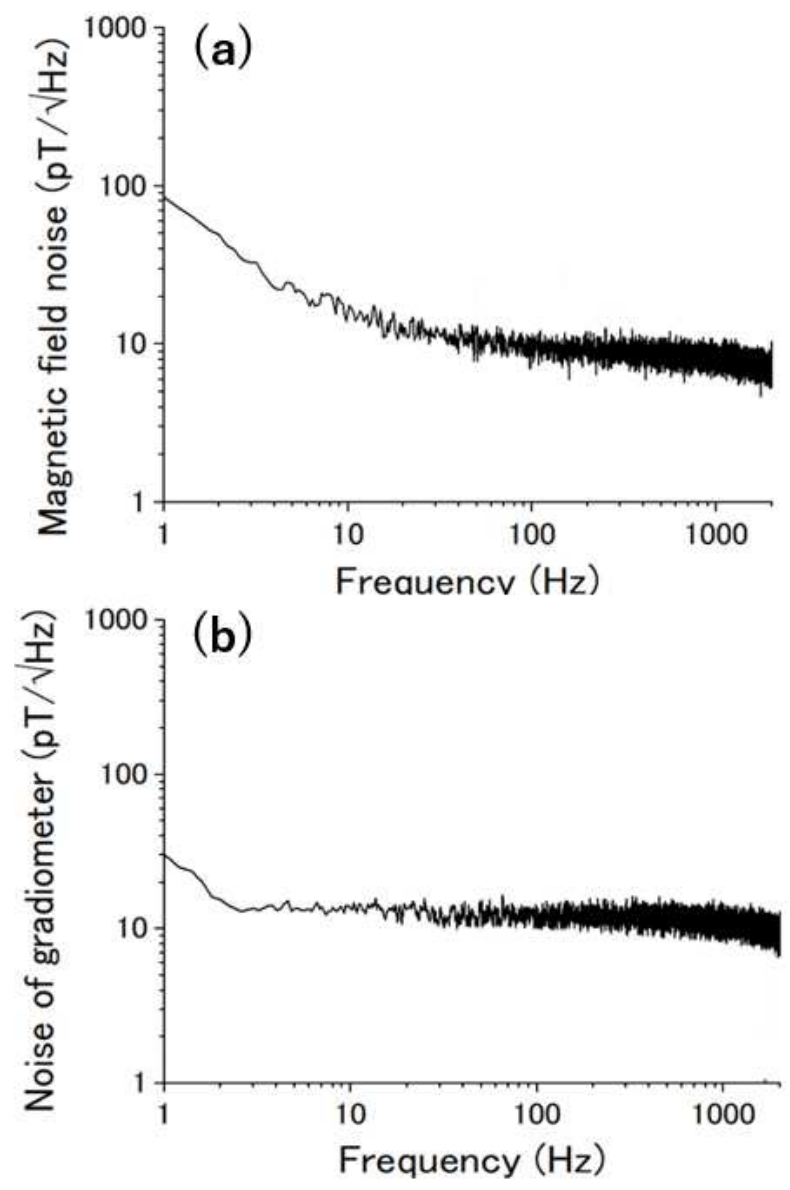

Figure 10. Magnetic field noise spectrum measured in a magnetic shielding box. (a). Noise spectrum measured by the magnetic sensor. (b). Noise spectrum measured by the gradiometer.

The magnetic field noise spectrum was also measured in unshielded environment. Figure 11 (a) shows the noise spectrum measured by the magnetic sensor. The peaks on the noise spectrum were $50 \mathrm{~Hz}$ interference and its harmonics. Figure 11 (b) shows the noise spectrum measured by the gradiometer. Not only the low frequency noise was reduced, bus also the $50 \mathrm{~Hz}$ interference was also reduced by about 30 times.
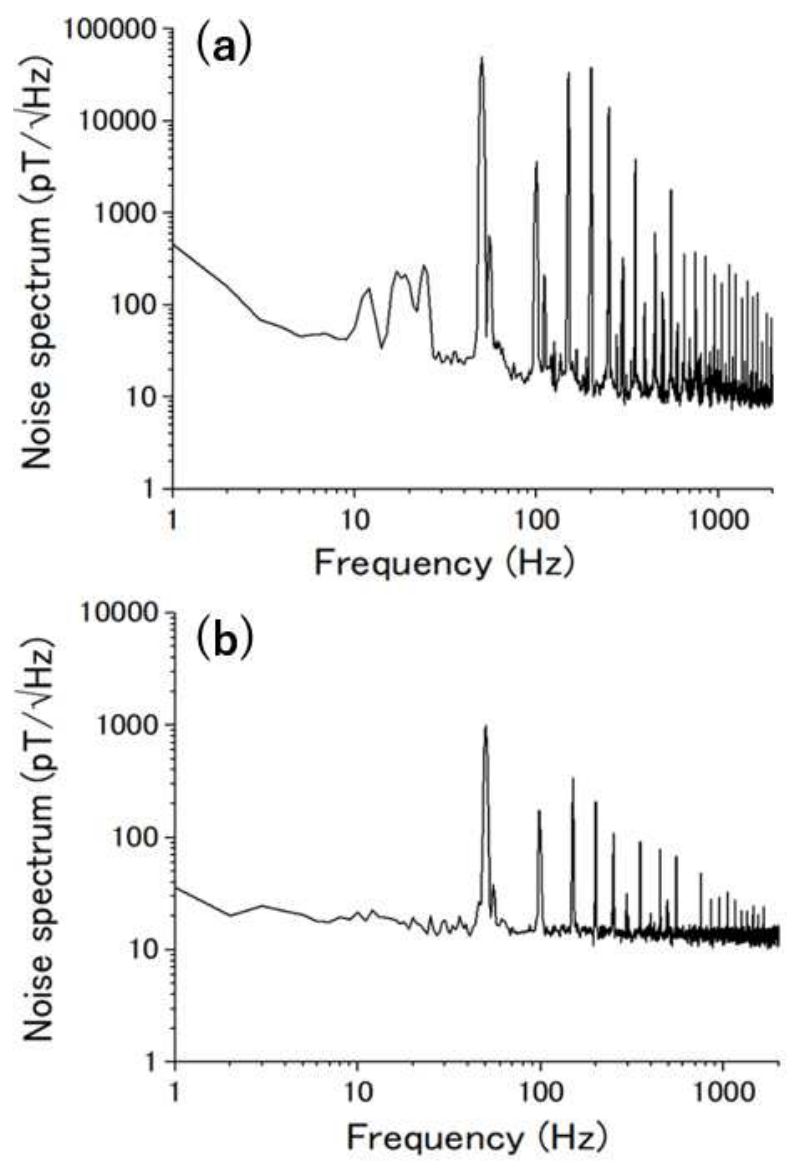

Figure 11. Magnetic field noise spectrum measured in an unshielded environment. (a). Noise spectrum measured by the magnetic sensor. (b). Noise spectrum measured by the gradiometer.

\section{Application System Using the Electrical Gradiometer of the Magnetic Sensor}

\subsection{Setup of the ECT System Using Gradiometer}

For the deep defect detection using eddy current testing (ECT) method, normally lower excitation frequency is used to get bigger penetration depth of eddy current. And the signal produced by deep defect is very small, so magnetic sensor or gradiometer with low noise at low frequency is needed. An eddy current testing (ECT) system was developed using the gradiometer. Figure 12 shows schematic block diagram of the ECT system. The excitation magnetic field was produced by a double-D coil with the diameter of $2 \mathrm{~cm}$ and 20 turns. The direction of the magnetic field produced by the double-D coil was vertical. To detect the deep defect, a lower excitation frequency of $170 \mathrm{~Hz}$ was used. The amplitude of the excitation current was about $100 \mathrm{~mA}$. The double-D coil was fixed to the bottom of the FeCoSiB magnetic sensor 1 of the gradiometer and the position was adjusted to make output of the gradiometer smallest. 


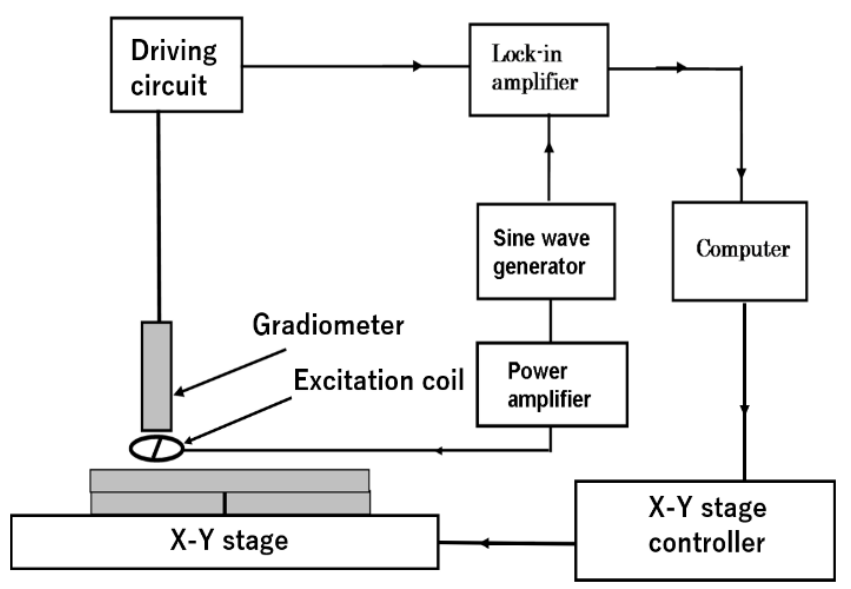

Figure 12. Setup of the eddy-current NDE system using gradiometer.

\subsection{Defect Detection Using the ECT System with the Gradiometer}

The ECT specimen was prepared using aluminum plates. A horizonal artificial crack defect with the length of about $1 \mathrm{~cm}$ was made in a $1 \mathrm{~mm}$ thick aluminum plate, and it was covered by another aluminum plate with the thickness of $8 \mathrm{~mm}$. An $\mathrm{X}-\mathrm{Y}$ stage was used for the scanning. The measurement was done without shielding. Figure 13 shows the signals produced by the defect when the scanning direction was perpendicular to the direction of the crack.

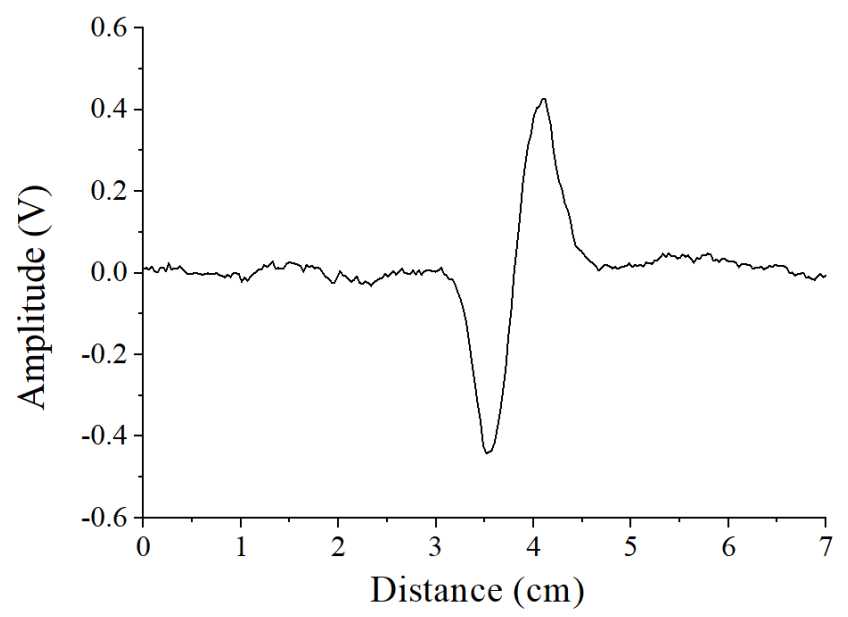

Figure 13. Signal of a crack defect with $8 \mathrm{~mm}$ depth detected by the electrical gradiometer composed of two $\mathrm{FeCoSiB}$ magnetic sensors.

\section{Discussion}

The achieved best magnetic field resolution of GMI magnetic sensor at white noise range was about $1 \mathrm{pT} / \sqrt{ } \mathrm{Hz}$ [24]. The $1 / \mathrm{f}$ noise was about $10 \mathrm{pT} / \sqrt{ } \mathrm{Hz}$ at $10 \mathrm{~Hz}$. Using the gradiometer composed of the GMI magnetic sensor with a pickup coil and a reference coil, they even tried to measure magnetoencephalogram (MEG) signals under no shield environment. For GMI magnetic sensor, the intrinsic magnetic field noise was estimated to be $8.4 \mathrm{fT} / \sqrt{ } \mathrm{Hz}$ [15], which is far smaller than the noise of a real GMI sensor. A reasonable estimation of $\mathrm{pT} / \sqrt{ } \mathrm{Hz}$ level was given [25].
Although the operation principle of the sensor in this paper is different from the normal GMI sensor, it is also very difficult to accurately estimate the intrinsic noise of this kind magnetic sensor. Here, a simple estimation was given. The impedance of the coil with the amorphous wire core was about $3 \Omega$ at $1 \mathrm{MHz}$. The thermal noise level was estimated to be about $\sqrt{ }\left(4 k_{B} T R\right) \approx 0.22 \mathrm{nV} / \sqrt{\mathrm{Hz}}$, where $k_{B}$ is the Boltzmann's constant, $T$ is the temperature, $R$ is the impedance of the coil with the amorphous wire core. The quality factor of the LC resonant circuit was about 10. After the LC resonance circuit, the noise was amplified to 2.2 $\mathrm{nV} / \sqrt{\mathrm{Hz}}$ and the resistance was amplified to $300 \Omega$. From the datasheet of $\mathrm{AD} 829$, its equivalent input voltage noise spectrum is about $1.7 \mathrm{nV} \sqrt{ } \mathrm{Hz}$ and the current noise spectrum is about $1.5 \mathrm{pA} / \sqrt{\mathrm{Hz}}$. Thus, the total noise was about $\sqrt{ }\left(2.2^{2}+1.7^{2}+(1.5 \times 0.3)^{2}\right) \approx 2.8 \mathrm{nV} \sqrt{\mathrm{Hz}}$. After the LC resonant circuit, the signal amplitude was about $100 \mathrm{mV} /$ Gauss, so the theoretical magnetic field noise spectrum of the magnetic sensor was estimated to be about $2.8 \mathrm{pT} / \sqrt{\mathrm{Hz}}$, which had the same order as that of a real magnetic sensor. Considering of the contributions of the DC bias current noise and AC bias current noise, this value was reasonable.

\section{Summary}

In a summery, the noise contribution of each part the magnetic sensor with the $\mathrm{FeCoSiB}$ amorphous wire was investigated. The bias current noise had the main contribution to the low frequency noise of the magnetic sensor. To reduce the low frequency noise, an electrical gradiometer composed of two magnetic sensors with the same bias DC current and AC current was developed. The results proved that the gradiometer was not only effective to reduce the low frequency noise caused the bias current, but also effective to reduce the influence of the line interference. An ECT system was constructed using the gradiometer and the deep defect with depth of about $8 \mathrm{~mm}$ in the aluminum plate was successfully detected.

\section{References}

[1] Mohri, K., Honkura, Y. (2007). Amorphous wire and CMOS IC based magneto-impedance sensors - origin, topics, and future. Sensor Letters, 5, 267-270.

[2] Uchiyama, T., Hamada, N., and Cai, C. (2014). Highly sensitive CMOS magnetoimpedance sensor using miniature multi-core head based on amorphous wire. IEEE Trans. Magn., 50,4005404 .

[3] Dolabdjian, C., Menard, D. (2017). Giant magneto-Impedance (GMI) magnetometers. High Sensitivity Magnetometers, edited by Grosz, A., Haji Sheikh, M. J., Mukhopadhyay, S. C. Title of series: Smart Sensors Measurement and Instrumentation, 19, 103-126.

[4] Weiss, E., Grosz, A., Paperno, E. (2015). Duty cycle operation of an orthogonal fluxgate. IEEE Sensors Journal, 15, 1977-1981. 
[5] Butta, M., Janosek, M. (2018). Very low frequency noise reduction in orthogonal fluxgate. AIP Advances, 8, 047203.

[6] Janosek, M., Butta, M., Dressler, M, Saunderson, E, Novotn, D., Fourie, C. J. (2020). 1-pT Noise Fluxgate Magnetometer for Geomagnetic Measurements and Unshielded Magnetocardiography. IEEE Trans. Instrum. Meas., 69, 2552-2560.

[7] Clarke, J., Braginski, A. I. (Eds.) (2004). Fundamentals and technology of SQUIDs and SQUID systems. In The SQUID Handbook. WILEY-VCH Verlag GmbH\&Co. KgaA: Weinheim, Germany, Volume 1, ISBN 3-52740229-2.

[8] Kominis, I. K., Kornack, T. W., Allred, J. C., Romalis, M. V. (2003). A subfemtotesla multichannel atomic magnetometer. Nature, 422, 596-599.

[9] Honkura, Y. (2012). The development of MI sensor and its applications to mobile phone. In Proceedings of the 2012 Advanced Electromagnetics Symposium (AES 2012), Paris, France, 16 April 2012.

[10] Postolache, O., Ramos, H. G., Ribeiro, A. Lopes. (2013). GMR array uniform eddy current probe for defect detection in conductive specimens. Measurement, 46, 4369-4378.

[11] He, D. F., Shiwa, M., Jia, J. P., Takatsubo, J., Moriya, S. (2011). Multi-frequency ECT with AMR sensor. NDT\&E International, 44, 438-441.

[12] Treutler, C. P. O. (2001). Magnetic sensors for automotive applications. Sens. Actuat. A Phys., 91, 2-6.

[13] Uehara, M., and Nakamura, N. (2007). Scanning magnetic microscope system utilizing a magneto-impedance sensor for a nondestructive diagnostic tool of geological samples. Review of Scientific Instruments, 78, 043708.

[14] Mahesh, Raja P., Kavithak, Arunachalam, and Krishnan Balasubramanian. (2016). Experimental validation of an eddy current probe for defect detection in thick conducting specimen. 42nd annual Review of Progress in Quantitative Nondestructive Evaluation (QNDE), together with the 6th European American Workshop on the Reliability of NDE, held at the Hyatt Regency Minneapolis, Minneapolis, Minnesota, July 26-31, 2015. AIP Conference Proceedings, 1706, 020025.
[15] Melo, L. G. C., Menard, D., Yelon, A., Ding, L., Saez, S. et al. (2008) Optimization of the magnetic noise and sensitivity of giant magnetoimpedance sensors. J. Appl. Phys., 103, 033903.

[16] Butta, M., Sasada, F. (2012). Sources of noise in a magnetometer based on orthogonal fluxgate. IEEE Trans. Magn., 48, 1508-1511.

[17] He, Dongfeng; and Shiwa, Mitsuharu. (2014). A magnetic sensor with amorphous wire. Sensors, 14, 10644-10649.

[18] He, Dongfeng. (2019). A feedback method to improve the dynamic range and the linearity of magneto-impedance magnetic sensor. Journal of Sensors, 19, 2413408.

[19] He, Dongfeng. (2020). PT-level high-sensitivity magnetic sensor with amorphous wire. Sensors, 20, 00161.

[20] Koch, R. H., Rozen, J. R., Sun, J. Z., and Gallagher, W. J. (1993). Three SQUID gradiometer. Appl. Phys. Lett., 63, 403-405.

[21] Sheng, D., Perry, A. R. et al. (2017). A microfabricated optically-pumped magnetic gradiometer. Appl. Phys. Lett., 110, 031106.

[22] Riveros, P. A. D., Silva, E. C., Pacheco, S., Cabrera, L. S. B., Barbosa, C. R. H. (2020). Design implementation and experimental characterization of a high sensitivity GMI gradiometer with an interference compensation system. Science Measurement \& Technology IET, 14, 688-694.

[23] https://www.ti.com/lit/wp/slyy134/slyy134.pdf, Measuring various types of low-frequency noise in DC/DC switching converters.

[24] Uchiyama, T., Mohri, K., Honkura, Y., Panina, L. V. (2012). Recent advances of pico-tesla resolution magneto-impedance sensor based on amorphous wire CMOS IC MI sensor. IEEE Transactions on Magnetics, 48, 3833-3839.

[25] Ménard, D., Rudkowskaa, G., Climea, L. Ciureanua, P., Yelona, A., Saezb, S., Dolabdjianc, C., Robbes, D. (2006). Progress towards the optimization of the signal-to-noise ratio in giant magnetoimpedance sensors. Sensors and Actuators A, $129,6-9$. 